\title{
Avaliação comparativa das incidências radiográficas cross-table e Ducroquet para a medição do ângulo alfa do quadril*
}

\section{Comparative Evaluation of Cross-table and Ducroquet Incidences for Measurement of the Hip Alpha Angle}

\author{
Giancarlo Cavalli Polesello ${ }^{1}$ (i) Marcelo Cavalheiro Queiroz ${ }^{1}$ Walter Ricioli Junior ${ }^{1}$ Marco Rudelli ${ }^{1}$ \\ Marcus Aurelius Araújo Nunes ${ }^{1}$ Denise Tokechi Amaral ${ }^{2}$
}

${ }^{1}$ Grupo de Quadril, Departamento de ortopedia e Traumatologia, Faculdade de Ciências Médicas da Santa Casa de São Paulo

Endereço para correspondência Giancarlo Cavalli Polesello, MD, PhD, (FCMSCSP), Irmandade Santa Casa de Misericórdia, São Paulo, Rua Dr. Cesário Mota Júnior, 112, Bairro Vila Buarque, São Paulo, SP, Brasil

${ }^{2}$ Serviço de Radiologia, Hospital Sírio Libanês, São Paulo, SP, Brasil SP, 01221-020, Brazil (e-mail: dot.quadril@hotmail.com).

Rev Bras Ortop 2019;54:428-433.

\section{Resumo \\ Palavras-chave \\ - impacto femoroacetabular \\ - quadril/ anormalidades \\ - quadril/diagnóstico por imagem \\ - radiografia}

Objetivo O objetivo do presente trabalho é comparar a equivalência da medida do ângulo alfa do quadril usando as incidências laterais de Ducroquet e de cross-table. Método Estudamos 90 pacientes, com um total de 95 quadris, e padronizamos a realização das radiografias conhecidas como "Ducroquet" e "cross-table." A incidência de perfil de cross-table foi realizada em $15^{\circ}$ de rotação interna com o paciente em posição supina, e a incidência de perfil de Ducroquet foi padronizada com o paciente posicionado em decúbito dorsal, em $90^{\circ}$ de flexão e $45^{\circ}$ de abdução do quadril. 0 ângulo alfa foi medido em ambas as radiografias de perfil, por dois radiologistas especializados em afecções musculoesqueléticas. As medidas foram realizadas em 2 épocas diferentes: uma avaliação inicial e outra após 4 semanas. O teste t de Student foi utilizado e calculou o coeficiente de correlação intraclasse (CCl).

Resultados Encontramos boa correlação intraobservador para ambas as incidências radiográficas em diferentes momentos. Não houve diferença estatisticamente significante entre as medidas feitas pelas duas visualizações. No entanto, a correlação interobservadores foi baixa.

Conclusão A incidência radiográfica de perfil de Ducroquet é uma boa opção para a medida do ângulo alfa e pode ser usada ao invés da incidência radiográfica de perfil cross-table.

Trabalho feito no Grupo do Quadril do Departamento de Ortopedia e Traumatologia da Irmandade Santa Casa de Misericórdia, Faculdade de Ciências Médicas da Santa Casa de São Paulo (FCMSCSP), São Paulo, SP, Brasil.

(D) Giancarlo Cavalli Polesello's ORCID is https://orcid.org/0000-00029524-8871.

recebido

05 de Março de 2019

aceito

22 de Janeiro de 2019
DOI https://doi.org/

$10.1055 / \mathrm{s}-0039-1693044$. ISSN 0102-3616.

\section{Introdução}

O impacto femoroacetabular (IFA) é uma entidade bem estabelecida na ortopedia mundial. ${ }^{1-6}$ Ele é considerado um dos principais processos de origem mecânica que levam à artrose do quadril. ${ }^{1,3,4,6-9}$ Existem parâmetros radiográficos bem

Copyright $\odot 2019$ by Sociedade Brasileira License terms de Ortopedia e Traumatologia. Published by Thieme Revnter Publicações Ltda, Rio de Janeiro, Brazil 


\section{Abstract}

\section{Keywords}

- femoracetabular impingement

- hip/abnormalities

- hip/diagnostic imaging

- radiography
Objective The purpose of the present paper is to compare the equivalence of the measurement of the $\alpha$ angle using the Ducroquet and cross-table lateral views.

Methods We have recruited 90 patients, resulting in 95 hips. We have standardized the realization of the radiographic views. The incidence of the lateral cross-table views were taken with $15^{\circ}$ of internal rotation with the patient in the supine position, and the incidence of the Ducroquet views was standardized with the patient in the supine position, with $90^{\circ}$ of flexion and $45^{\circ}$ of abduction of the hip. The $\alpha$ angle was measured in both lateral views, by two musculoskeletal radiologists. The measurements were performed in 2 different times: an initial evaluation and another 4 weeks afterwards. The $t$ Student test was used and calculated the intraclass correlation coefficient (ICC). Results We have found a good intraobserver correlation for both views in different times; there was no statistically significant difference between the measurements performed by the two views. However, the interobserver correlation was low.

Conclusion In conclusion, the Ducroquet profile view is a good choice for the $\alpha$ angle measurement and can be used instead of the cross-table view. definidos para seu diagnóstico. ${ }^{2,4,10}$ As alterações morfológicas desta afecção não são, em sua maioria, visíveis na radiografia anteroposterior da bacia. ${ }^{2-4}$ As incidências em perfil demonstram melhor a porção não esférica do colo femoral e as deformidades causadas pelo IFA. , $^{8,9,11-13}$

A medida desta porção não esférica da junção cabeça-colo femoral pode ser medida com o ângulo alfa (ângulo de Nötzli). ${ }^{8,12} \mathrm{O}$ ângulo alfa é formado por uma linha traçada através do centro da cabeça femoral, seguindo pelo eixo do colo femoral, e por outra linha que liga o centro da cabeça femoral até o ponto em que ocorre a protrusão da cabeça femoral além do círculo desenhado em torno da cabeça femoral (ponto este onde termina a esfericidade da cabeça femoral) ${ }^{8,12} \mathrm{Na}$ descrição original, o ângulo foi medido na ressonância magnética (RM) em corte axial oblíquo. ${ }^{8}$

Várias incidências em perfil foram descritas para investigar anormalidades do colo femoral, mas a mais comumente utilizada é a incidência em perfil cross-table. ${ }^{5,6,14}$ A incidência de perfil Ducroquet é outra opção, fácil de fazer, independentemente da mesa, e requer somente $90^{\circ}$ de flexão e de 30 a $45^{\circ}$ de abdução. ${ }^{12}$ Por sua facilidade de realização técnica, incluindo intraoperatoriamente na artroscopia do quadril, é nossa escolha de imagem para avaliar o ângulo alfa.

O objetivo do presente trabalho é comparar a equivalência da medida do ângulo alfa usando as incidências de perfil Ducroquet e cross-table.

\section{Materiais e Métodos}

Trata-se de um estudo prospectivo feito no Departamento de Ortopedia e Traumatologia da Irmandade da Santa Casa de Misericórdia de São Paulo no período de janeiro de 2008 a abril de 2010. Foram recrutados 90 pacientes, resultando em 95 quadris, selecionados voluntariamente e submetidos a radiografias de perfil Ducroquet e cross-table. O consentimento informado foi obtido de todos os pacientes, com a aprovação do número do Conselho de Revisão Institucional (240/09).

\section{Radiografias}

Padronizamos a realização das incidências radiográficas. Todas foram realizadas pelos mesmos técnicos, com a mesma técnica, e supervisionada por um ortopedista. A incidência em perfil cross-table (-Figs. 1A e 1B) foi realizada com 0 paciente em posição supina com $15^{\circ}$ de rotação interna do membro inferior estudado e flexão de $90^{\circ}$ do membro contralateral para evitar interposição de imagem, como preconizado pela técnica, e a incidência de Ducroquet (-Figs. 1C e 1D) foi padronizada com o paciente posicionado em decúbito dorsal, com $90^{\circ}$ de flexão e $45^{\circ}$ de abdução do quadril afetado, em rotação neutra.

\section{Medição Radiográfica}

O ângulo alfa (ângulo de Nötzli) ${ }^{8,12}$ foi medido em ambas as incidências de perfil, por dois radiologistas musculoesqueléticos, usando as mesmas técnicas de medição. 8,130 ângulo foi medido digitalmente com o software Image, versão 1.37 (National Institutes of Health, Bethesda, MD, EUA).

As medidas foram realizadas em duas épocas diferentes: avaliação inicial (chamada "pré") e outra realizada 4 semanas depois (denominada "pós”). A análise estatística foi feita com resumo de variáveis, gráficos boxplot e diagramas de dispersão. O teste t de Student foi utilizado e calculou-se o coeficiente de correlação intraclasse (CCI) com o software PASW Statistics for Windows, Versão 10.0 (SPSS Inc., Chicago, IL, EUA). O nível de significância foi determinado como sendo de 0,05 (5\%).

\section{Resultados}

As medidas do ângulo alfa foram semelhantes para cada observador, tanto em incidências tomadas em momentos diferentes: pré e após 4 semanas (-Fig. 2 e -Tabela 1).

A análise foi realizada:

A) Comparando-se os resultados entre os observadores 1 e 2 obtidos para cada incidência radiográfica na $1^{\underline{a}}$ e na $2^{\underline{a}}$ avaliação (pré e pós). 


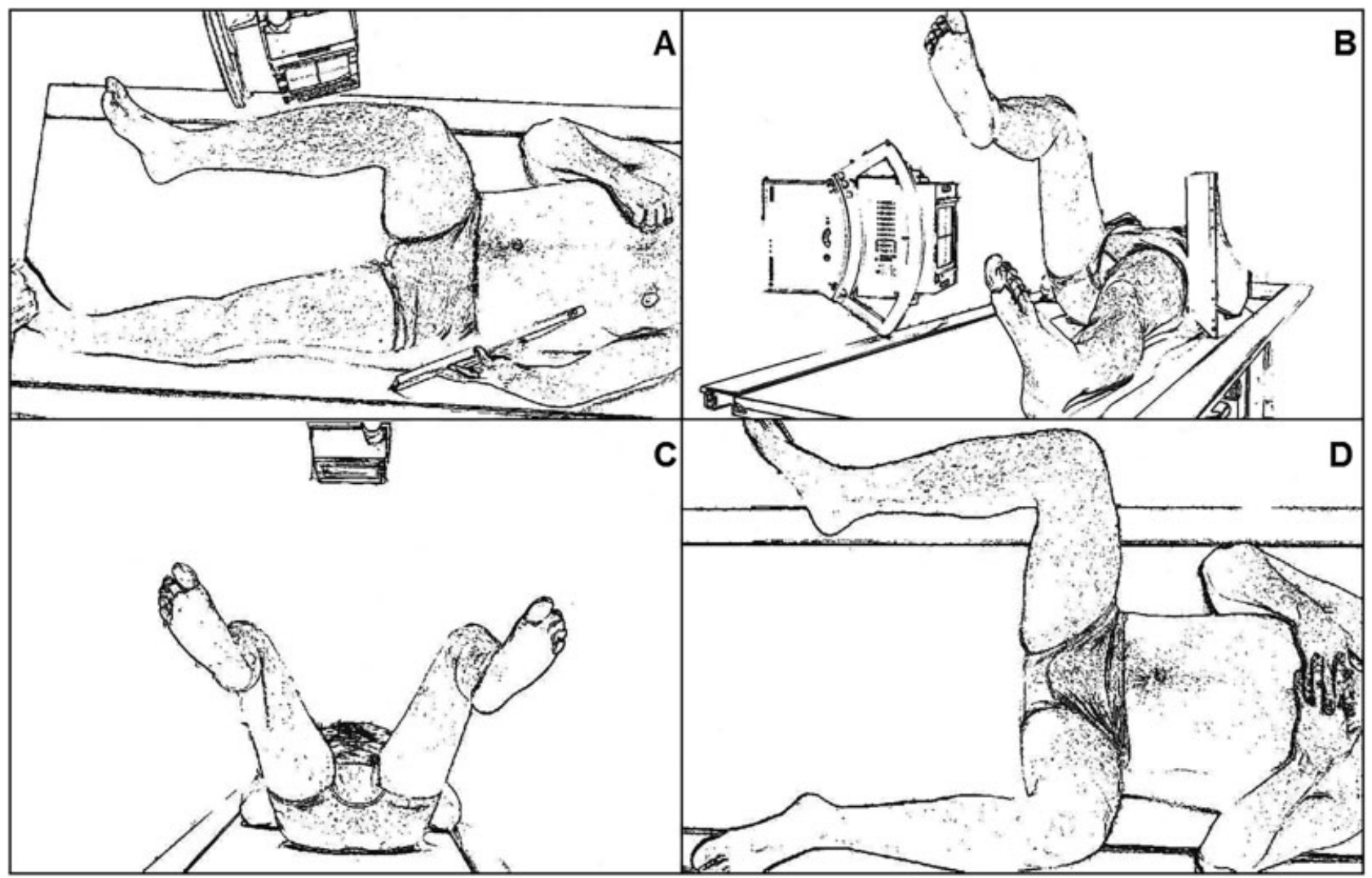

Fig. 1 (A e B). Posição para realizar a incidência de perfil cross-table; (C e D). Posição para realizar a incidência de perfil de Ducroquet.

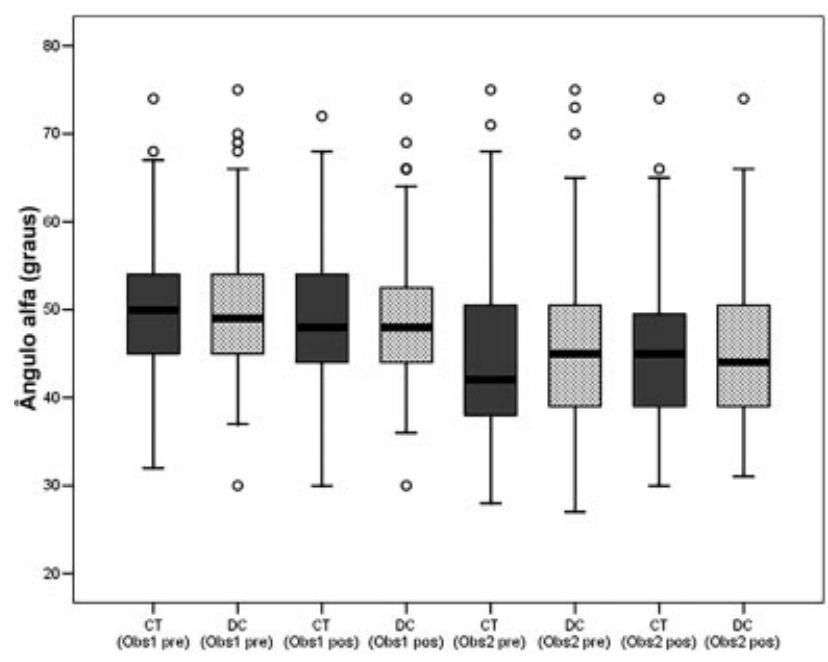

Fig. 2 Gráfico Boxplot das medidas do ângulo alfa do quadril feitas por dois observadores diferentes, em duas vistas diferentes (Ducroquet e cross-table) em dois momentos diferentes (pré e pós). Abreviações: CT: cross-table; DC: Ducroquet view; Obs1: observador 1; Obs2: observador 2; Pre: medições iniciais; Pos: medições após 4 semanas.

Quanto à análise entre observadores:

A.1) Cross-table: houve disparidade entre os observadores 1 e 2 na pré-avaliação ( - Tabela 2 e - Fig. 3A) e na pós-avaliação (-Tabela 2 e - Fig. 3B).

A.2) Ducroquet: houve disparidade entre os observadores 1 e 2 na pré-avaliação ( - Tabela 2 e -Fig. 3C) e na pós-avaliação (-Tabela 2 e - Fig. 3D).
Tabela 1 Medições radiográficas do ângulo alfa do quadril por dois observadores diferentes, em duas incidências diferentes (Ducroquet e cross-table) em dois tempos diferentes (pré e pós)

\begin{tabular}{|l|l|l|l|l|}
\hline Observador & $\begin{array}{l}\text { Incidência } \\
\text { radiográfica }\end{array}$ & Tempo & $\begin{array}{l}\text { Média(graus) } \\
\pm \text { DP }\end{array}$ & $\begin{array}{l}\text { Mediana } \\
\text { (graus) }\end{array}$ \\
\hline 1 & cross-table & pre & $49,8 \pm 7,43$ & 50,1 \\
\hline & & pos & $49 \pm 7,31$ & 48,5 \\
\hline 1 & Ducroquet & pre & $50,2 \pm 8,32$ & 49,8 \\
\hline & & pos & $48 \pm 7,89$ & 48,2 \\
\hline & cross-table & pre & $44,9 \pm 9,77$ & 42,3 \\
\hline 2 & & pos & $45,4 \pm 8,68$ & 45,5 \\
\hline & Ducroquet & pre & $45,2 \pm 9,22$ & 45,2 \\
\hline & & pos & $44,9 \pm 7,88$ & 44,4 \\
\hline
\end{tabular}

Abreviações: DP, desvio padrão; Pos: medições após 4 semanas; Pre: medições iniciais.

Houve diferença estatisticamente significante $(p=0.000)$ para as medidas entre observadores para ambas as incidências nas 2 épocas:

B) Comparando a correlação das medidas de cross-table e de Ducroquet para cada observador em ambas as avaliações de tempo.

B.1) Quanto aos observadores 1 e 2, houve pouca disparidade nas medidas do ângulo alfa do quadril em ambas as incidências ao mesmo tempo ( - Tabela 2 e - Fig. 4A): 
Tabela 2 Coeficientes de correlação intraclasse das medidas do ângulo alfa do quadril feitas por dois observadores nas incidências cross-table e Ducroquet

\begin{tabular}{|l|l|l|}
\hline Análise & Coeficiente de correlação intraclasse & IC $95 \%$ \\
\hline CT Obs1 (pre) x CT Obs2 (pre) & 0,588 & $0,381-0,726$ \\
\hline CT Obs1 (pos) x CT Obs2 (pos) & 0,653 & $0,397-0,790$ \\
\hline DC Obs1 (pre) x DC Obs2 (pre) & 0,578 & $0,226-0,754$ \\
\hline DC Obs1 (pos) x DC Obs2 (pos) & 0,718 & $0,405-0,847$ \\
\hline CT Obs1 (pre) x DC Obs1 (pre) & 0,938 & $0,907-0,959$ \\
\hline CT Obs1 (pos) x DC Obs1 (pos) & 0,969 & $0,954-0,979$ \\
\hline CT Obs2 (pre) x DC Obs2 (pre) & 0,862 & $0,793-0,908$ \\
\hline CT Obs2 (pos) x DC Obs2 (pos) & 0,945 & $0,918-0,964$ \\
\hline
\end{tabular}

Abreviações: CT: cross-table; DC: Ducroquet; IC, intervalo de confiança; Obs1: Observador 1; Obs2: Observador 2; Pos: medições após 4 semanas; Pre: medições iniciais.

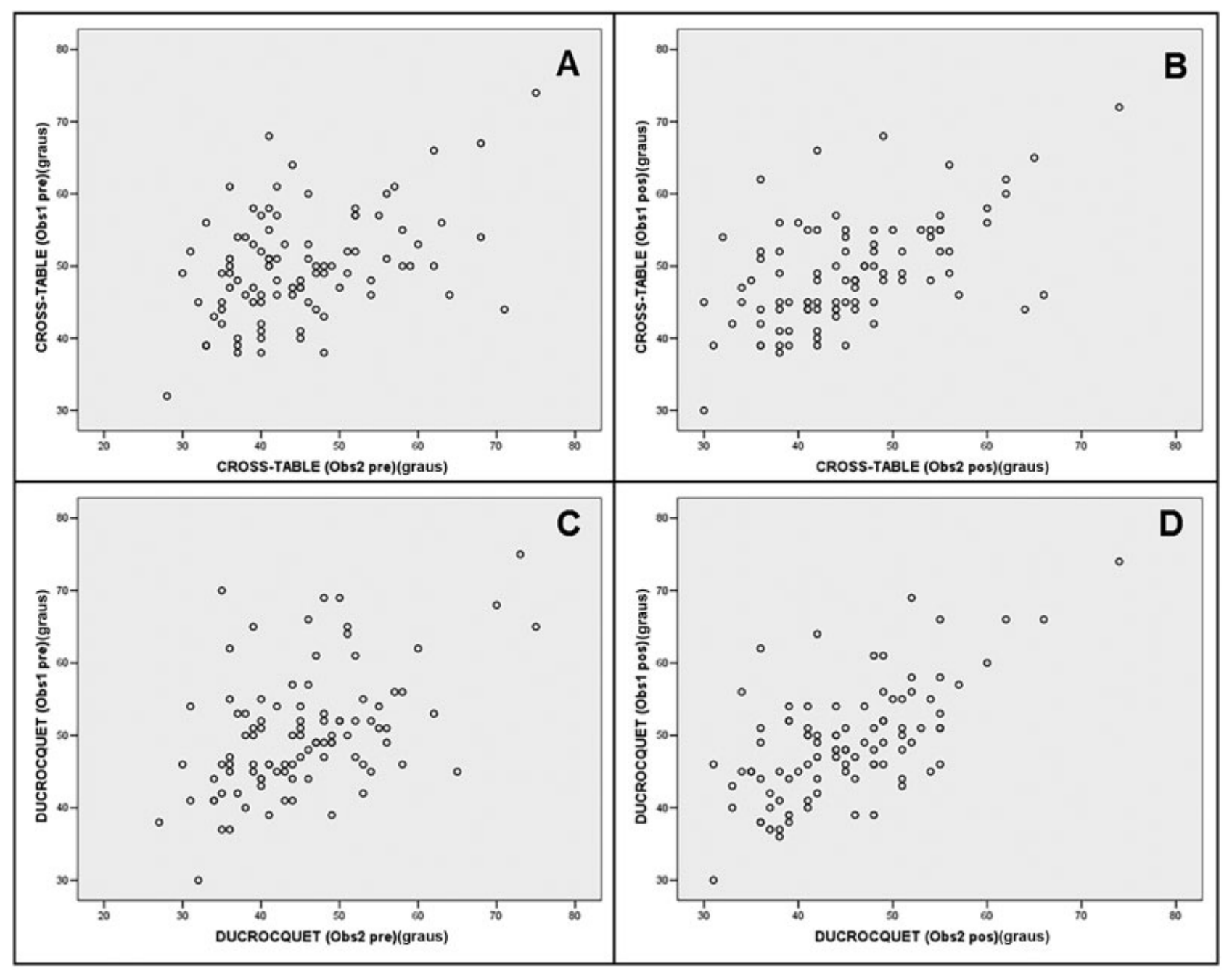

Fig. 3 (A) Correlação das medidas iniciais do ângulo alfa do quadril feitas por dois observadores diferentes na visão de cross-table. Pre: medições iniciais; (B) Correlação das segundas medidas do ângulo alfa do quadril feitas por dois observadores diferentes na visão de cross-table. Pos: medições após 4 semanas; (C) Correlação das medidas iniciais do ângulo alfa do quadril feitas por dois observadores diferentes na visão de Ducroquet. Pre: medições iniciais;

(D) Correlação das segundas medições do ângulo alfa do quadril feitas por dois observadores diferentes na vista Ducroquet. Pre: medições iniciais.

observador 1, $1^{\text {a }}$ medida, - Tabela 2 e - Fig. 4B: observador 1, 2⿺ medida, - Tabela 2 e - Fig. 4C: observador 2 , $1^{\text {a }}$ medida, - Tabela 2 e - Fig. 4D: medição do observador de 2 segundos.
Não houve diferença estatisticamente significativa $(p>0,05)$ no valor do ângulo alfa medido em ambas as incidências radiográficas pelo mesmo observador ao mesmo tempo (-Tabela $\mathbf{3}$ ). 


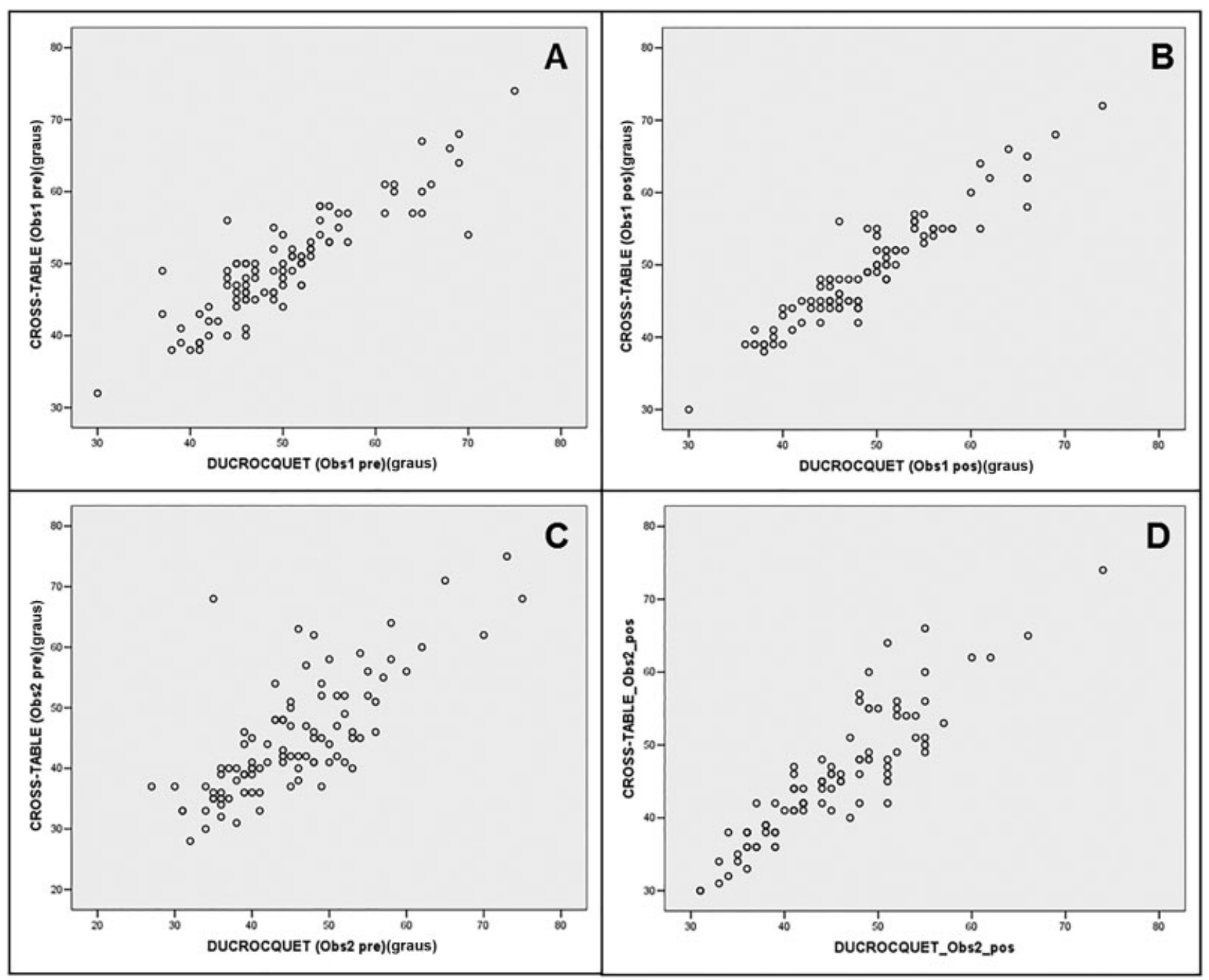

Fig. 4 (A) Correlação das medidas iniciais do ângulo alfa do quadril feitas pelo observador 1 nas vistas cross-table e Ducroquet. Pre: medições iniciais. Obs1: observador 1; (B) Correlação das segundas medições do ângulo alfa do quadril feitas pelo observador 1 nas vistas Cross-table e Ducroquet. Pos: segundas medições. Obs1: Observador 1.; (C) Correlação das medidas iniciais do ângulo alfa quadril feitas pelo observador 2 nas incidências cross-table e Ducroquet. Pre: medições iniciais. Obs2: observador 2; (D) Correlação das segundas medições do ângulo alfa do quadril feitas pelo observador 2 nas vistas cross-table e Ducroquet. Pos: segundas medições. Obs2: Observador 2.

Tabela 3 Análise intraobservador das medidas do ângulo alfa do quadril em duas visualizações diferentes (Ducroquet e cross-table) em dois momentos diferentes (pré e pós). Pré: medições iniciais. Pós: medições após 4 semanas

\begin{tabular}{|l|l|l|}
\hline Análise & & valor-p \\
\hline $\mathbf{1}$ & $\begin{array}{l}\text { cross-table (Obs1 pre) - } \\
\text { Ducrocquet (Obs1 pre) }\end{array}$ & 0,309 \\
\hline $\mathbf{2}$ & $\begin{array}{l}\text { cross-table (Obs2 pre) - } \\
\text { Ducrocquet (Obs2 pre) }\end{array}$ & 0,611 \\
\hline $\mathbf{3}$ & $\begin{array}{l}\text { cross-table (Obs1 pos) - } \\
\text { Ducrocquet (Obs1 pos) }\end{array}$ & 0,699 \\
\hline 4 & $\begin{array}{l}\text { cross-table (Obs2 pos) - } \\
\text { Ducrocquet (Obs2 pos) }\end{array}$ & 0,223 \\
\hline
\end{tabular}

Abreviações: Pos: medições após 4 semanas; Pre: medições iniciais; Obs1: Observador 1; Obs 2: Observador 2.

\section{Discussão}

Comparando a mensuração do ângulo alfa por dois avaliadores através das incidências radiográficas Ducroquet e cross-table, observamos boa correlação intraobservador, porém baixa correlação interobservador.

O diagnóstico de alterações sutis que podem causar coxartrose precoce do quadril está tendo ótimo desenvolvimento nos últimos anos. ${ }^{15}$ As radiografias são valiosas para a detecção de mudanças morfológicas femorais e acetabulares. ${ }^{4,11,12}$

O ângulo alfa (ângulo de Nötzli) é frequentemente usado para diagnosticar deformidades patológicas do colo femoral. $.8,8,12,13,16$

Existem controvérsias quanto à incidência ideal para medir o ângulo alfa. Meyer et al ${ }^{13}$ compararam 6 radiografias (anteroposterior, Dunn, Dunn com $45^{\circ}$ de flexão, cross-table com $15^{\circ}$ de rotação interna, cross-table em rotação neutra, e cross-table $\operatorname{com} 15^{\circ}$ de rotação externa). A conclusão foi que a incidência de 
Dunn em $45^{\circ}$ ou $90^{\circ}$ de flexão ou a incidência cross-table com rotação interna são melhores para detectar alterações na esfericidade da transição cabeça-colo femoral, acrescentando que as incidências anteroposterior e cross-table com rotação externa não são capazes de detectar essas mudanças.

O perfil de Ducroquet é fácil de executar e representa o perfil verdadeiro da extremidade proximal do fêmur, mostrando perfeitamente a transição entre o colo e a cabeça femoral. ${ }^{11,12}$ Além disso, esta incidência é útil para controle intraoperatório das osteoplastias femorais, pois permite realizar imagens em perfil sem a necessidade do mover o braço da fluoroscopia.

A incidência radiográfica do perfil de cross-table também oferece visão em perfil verdadeiro do colo femoral, além de visão acessória do acetábulo, porém é tecnicamente mais exigente devido à necessidade de tubo intensificador de imagem móvel. Requer também mesa ortopédica que não interfira na altura do tubo. ${ }^{11,12}$ Muitos centros de ortopedia no Brasil não possuem aparelho móvel de raio-X ou mesa ortopédica adequada, os quais são necessários para fazer este tipo de incidência.

Observamos que houve diferença estatisticamente significante $(p=0,00)$ para as medidas interobservadores para ambas as incidências estudadas nas 2 épocas que foram realizadas.

As medições médias do observador 2 foram inferiores às do observador 1 em todas as medidas, o que pode ser devido a diferenças sutis nas técnicas de medição dos diferentes observadores.

Houve boa correlação intraobservador para ambas as incidências estudadas em diferentes épocas, onde não houve diferença estatisticamente significativa $(p=0,309$; $p=0,611 ; p=0,699 ; p=0,223$ ) entre as medidas feitas pelas 2 visualizações.

A análise dos parâmetros radiográficos realizados em diferentes momentos já foi relatada na literatura. ${ }^{1,13}$ Meyer et $\mathrm{al}^{13}$ mostraram que o uso de cross-table e de Dunn em posicionamento de membros em $45^{\circ}$ e $90^{\circ}$ (semelhante ao Ducroquet) reproduziu melhor a medida do ângulo alfa.

Encontramos no presente estudo que a medida do ângulo alfa era dependente do observador. Entretanto, esta disparidade não tende a existir entre o mesmo observador que realiza diferentes medidas ao longo de um determinado período.

Uma das limitações do nosso trabalho foi que tivemos dificuldades em fazer a incidência cross-table em pacientes obesos; naquele momento, nós só possuíamos máquinas convencionais de raios-X. Recentemente; temos máquinas digitais de raios-X disponíveis e achamos mais fácil gerenciar estas imagens em tais pacientes. Talvez a dificuldade na realização de perfil de cross-table seja devido à padronização da visão de Ducroquet em nosso hospital como a incidência radiográfica de escolha para visualizar o perfil do quadril. Outra limitação foi a análise por apenas dois radiologistas como observadores.

Dados estes resultados, acreditamos que a incidência radiográfica perfil de Ducroquet é uma boa opção para a medida do ângulo alfa, e pode ser usada sendo a incidência em perfil de escolha, pois pode ser feita facilmente durante artroscopias de quadril sem mover o braço da fluoroscopia.

\section{Conclusão}

Concluímos que há equivalência para a medição do ângulo alfa do quadril quando usamos as incidências radiográficas de cross-table e de Ducroquet.

\section{Conflitos de Interesses}

Os autores declaram não haver conflitos de interesses.

Agradecimentos

Agradecemos ao Prof. Daniel K. Scheffer pela análise estatística.

\section{Referências}

1 Clohisy JC, Carlisle JC, Trousdale R, Kim YJ, Beaule PE, Morgan P, et al. Radiographic evaluation of the hip has limited reliability. Clin Orthop Relat Res 2009;467(03):666-675

2 Fadul DA, Carrino JA. Imaging of femoroacetabular impingement. J Bone Joint Surg Am 2009;91(Suppl 1):138-143

3 Dudda M, Albers C, Mamisch TC, Werlen S, Beck M. Do normal radiographs exclude asphericity of the femoral head-neck junction? Clin Orthop Relat Res 2009;467(03):651-659

4 Tannast M, Siebenrock KA, Anderson SE. Femoroacetabular impingement: radiographic diagnosis-what the radiologist should know. AJR Am J Roentgenol 2007;188(06):1540-1552

5 Eijer H, Leunig M, Mahomed MN, Ganz R. Cross- table lateral radiographs for screening of anterior femoral head- neck offset in patients with femoro- acetabular impingement. Hip Int 2001;11 (01):37-41

6 Neumann M, Cui Q, Siebenrock KA, Beck M. Impingement-free hip motion: the 'normal' angle alpha after osteochondroplasty. Clin Orthop Relat Res 2009;467(03):699-703

7 Fraitzl CR, Käfer W, Nelitz M, Reichel H. Radiological evidence of femoroacetabular impingement in mild slipped capital femoral epiphysis: a mean follow-up of 14.4 years after pinning in situ. J Bone Joint Surg Br 2007;89(12):1592-1596

8 Nötzli HP, Wyss TF, Stoecklin CH, Schmid MR, Treiber K, Hodler J. The contour of the femoral head-neck junction as a predictor for the risk of anterior impingement. J Bone Joint Surg Br 2002;84 (04):556-560

9 Clohisy JC, Nunley RM, Otto RJ, Schoenecker PL. The frogleg lateral radiograph accurately visualized hip cam impingement abnormalities. Clin Orthop Relat Res 2007;462(462): $115-121$

10 Ito K, Minka MA II, Leunig M, Werlen S, Ganz R. Femoroacetabular impingement and the cam-effect. A MRI-based quantitative anatomical study of the femoral head-neck offset.J Bone Joint Surg Br 2001;83(02):171-176

11 Godefroy D, Chevrot A, Morvan G, Rousselin B, Sarazin L. [Plain films of pelvis]. J Radiol 2008;89(5 Pt 2):679-690, quiz 691

12 Chrétien J. -Lateral views of the hip-. Ann Radiol (Paris) 1997;40 (01):5-10

13 Meyer DC, Beck M, Ellis T, Ganz R, Leunig M. Comparison of six radiographic projections to assess femoral head/neck asphericity. Clin Orthop Relat Res 2006;445(445):181-185

14 Loder RT. Effect of femur position on the angular measurement of slipped capital femoral epiphysis. J Pediatr Orthop 2001;21(04): 488-494

15 Gosvig KK, Jacobsen S, Palm H, Sonne-Holm S, Magnusson E. A new radiological index for assessing asphericity of the femoral head in cam impingement. J Bone Joint Surg $\mathrm{Br} 2007 ; 89(10)$ : 1309-1316

16 Rakhra KS, Sheikh AM, Allen D, Beaulé PE. Comparison of MRI alpha angle measurement planes in femoroacetabular impingement. Clin Orthop Relat Res 2009;467(03):660-665 